Genre et filiation : pratiques et représentations

\title{
La langue japonaise est-elle la « mère » des Zainichi ?
}

Mythe et politique de la langue maternelle chez quelques écrivains zainichi

Ayame HOSOI

\section{(2) OpenEdition}

Journals

Édition électronique

URL : http://journals.openedition.org/transtexts/490

DOI : $10.4000 /$ transtexts.490

ISSN : 2105-2549

Éditeur

Gregory B. Lee

Référence électronique

Ayame HOSOI, «La langue japonaise est-elle la « mère » des Zainichi? », Transtext(e)s Transcultures 跨 文本跨文化 [En ligne], 8 | 2013, mis en ligne le 02 décembre 2013, consulté le 15 novembre 2019. URL : http://journals.openedition.org/transtexts/490; DOI : 10.4000/transtexts.490

(c) Tous droits réservés 


\section{Transtext(e)s}

Transcultures 跨文本跨文

化

Journal of Global Cultural Studies

$8 \mid 2013$ :

Genre et filiation : pratiques et représentations

Langage et parenté, langue maternelle, question du nom

\section{La langue japonaise est-elle la " mère » des Zainichi ?}

\section{Mythe et politique de la langue maternelle chez quelques écrivains zainichi}

\section{AyAme HOSOI}

\section{Résumés}

Français English

Chez les ressortissants coréens établis au Japon, appelés Zainichi, la langue se situe au centre de la question existentielle. Notre travail se focalise sur le problème de la langue maternelle ; ainsi, nous interrogeons le mode selon lequel se manifeste la langue dite maternelle au travers de quelques ouvrages littéraires. Par là, nous interrogeons le concept lui-même de « langue maternelle » dans le contexte de la modernité et tentons d'exposer le schéma politicoidéologique dans lequel la langue maternelle des Zainichi est perçue comme une " mère » à double-face. Tandis que, parallèlement, il nous apparaît qu'une autre langue maternelle, ou plutôt un générateur de la langue, se lit entre les lignes. Ce générateur que l'on ne peut identifier est présenté comme une sorte d'impossibilité de s'exprimer chez certains écrivains. $\mathrm{Ne}$ pourrions-nous pas trouver dans cette impossibilité la véritable tâche, la raison d'être, de la littérature?

For the Korean immigrants in Japan called Zainichi, language occupies the centre of an existential question. This work focuses on the problem of the (so-called) mother tongue. Hence, we shall examine the way this mother tongue manifests itself through some literary works. Through such an analysis, we question the concept itself of "mother tongue" in the context of modernity and try to reveal the politico-historical schema in which the Zainichi's mother tongue is perceived as a bicephalous mother. In the meanwhile it seems to us that in parallel another mother tongue, or rather a generator of language can be heard behind the words. For some 
writers, this unidentifiable generator appears like a kind of impossibility to express themselves. Couldn't we find in this very impossibility the real task, the real meaning of the existence of literature?

\section{Texte intégral}

Certaines œuvres produites par la population issue des ressortissants coréens établis au Japon - nous appelons cette population Zainichi - formèrent, au milieu des années 1960, un nouveau courant littéraire, appelé « littérature zainichi ». ${ }^{1}$ La définition la plus répandue de cette catégorie est donc la suivante : une littérature réalisée en langue japonaise par les écrivains zainichi. Mais, la question concernant la langue chez les Zainichi est bien plus complexe qu'elle ne le paraît dans cette définition.

Chaque écrivain zainichi se comporte d'une manière différente face à la situation linguistique imposée pendant la colonisation. Par exemple, même chez les Zainichi de la première génération, qui ont tous subi la politique d'homogénéisation linguistique pendant l'occupation japonaise, les comportements ne sont pas les mêmes avec la langue du colonisateur. Les réactions opposées des deux prédécesseurs représentatifs des écrivains zainichi, Jang Hyeok-ju (1905-1997) et Kim Sa-ryang (1914-1950), nous montre une réalité complexe de la relation avec les langues de ces écrivains. Jang Hyeok-ju choisi d'écrire en japonais pour une raison pratique, tandis que Kim Sa-ryang choisi, après avoir publié quelques œuvres en japonais malgré son animosité pour cette langue, de rentrer dans sa « patrie » (en République populaire démocratique de Corée) peu après la libération de la Corée et y poursuit sa création littéraire en coréen. ${ }^{2}$ D'autres encore écrivent en japonais, malgré leur profonde gêne vis-à-vis de cette langue. Par exemple, pour Kim Seok-beom (1925-), il lui est impossible d'échapper à une opération de traduction en japonais pour transcrire son univers artistique. Ce désaccord entre l'univers intérieur de l'individu et son expression vers l'extérieur est un dilemme mortifère pour un écrivain. Kim Seok-beom nomme «ことばの呪縛 (ensorcellement de la langue) » cette contradiction qui fait souffrir les écrivains zainichi dans leur création littéraire. ${ }^{3}$ En revanche, Kim Si-jong (1925-), qui est né et a grandi en Corée sous l'occupation japonaise, est tout à fait conscient que la langue japonaise continue à exister en lui comme la langue dominante. En se trouvant chassé de cette langue lors de la libération de la Corée, à l'âge de 17 ans, Kim Si-jong a tenté de réveiller son ethnicité coréenne par l'apprentissage de la langue coréenne. Mais à aucun moment il ne peut nier la présence de la langue japonaise, la langue acquise dans son enfance et la langue qui forme sa conscience intérieure, vis-à-vis du monde extérieur. "Sans me détacher de cette langue (= le japonais), je n'obtiendrai jamais une véritable libération personnelle (de la colonisation) ", avoue-t-il. ${ }^{4} \mathrm{Si}$ se libérer de cette langue de l'ancien colonisateur est synonyme de la véritable décolonisation pour certains Zainichi (notamment ceux de la première génération), regagner la langue coréenne est considéré comme une condition primordiale de l'affirmation de l'identité pour les jeunes Zainichi. Mais, quelle que soit la génération, nous pouvons affirmer que le problème de la langue occupe une place centrale dans l'écriture zainichi.

En outre, nous pourrions considérer cette littérature zainichi doublement " masculine ». Car, premièrement, une grande majorité de ses écrivains était, jusque dans les années 1970 environ, masculine. ${ }^{5}$ La voix des femmes, exclues du domaine intellectuel, y était absente. Deuxièmement, car dans un contexte postcolonial, la langue de création littéraire est celle de l'Etat-Nation japonais pour les Zainichi, demeurant dans une relation de type coloniale où le Japon peut-être représenté métaphoriquement par l'homme. Nous aimerions examiner ici ce deuxième aspect (c'est-à-dire, les images genrées des langues, en particulier celles dites « maternelle » et « non-maternelle »). 
Après avoir esquissé brièvement le thème de la " genderisation » linguistique dans certaines œuvres littéraires, nous tenterons de dévoiler le mythe de la langue maternelle sur laquelle s'appuient certains écrivains zainichi.

\section{La représentation genrée des langues chez les Zainichi}

En premier lieu, nous aimerions attirer l'attention sur l'intervention du concept de genre, autrement dit, la « genderisation » concernant les langues des Zainichi. Selon Igeta Midori, la structure de "genderisation » est basée sur une relation de pouvoir et de domination qui existe quelle que soit la civilisation, quelle que soit l'endroit géographique et quelle que soit l'époque. ${ }^{6}$ Sakai Naoki fait remarquer également que dans les films dont le thème principal est la relation amoureuse entre deux personnages de nationalité et/ou issus de groupes ethno-géographiques différents, la relation intime et personnelle pourrait faire office d'allégorie de la relation de la domination historique et géopolitique. ${ }^{7}$ Et, quasiment sans exception, les personnages masculin et féminin sont respectivement symboles du dominant et de la dominée. Une remarque similaire est aussi faite par l'intellectuel zainichi, Kang Sang-jung. ${ }^{8}$ Nous pouvons observer dans certaines œuvres de la littérature zainichi également cette représentation du dominant par les personnages masculins. Ainsi, lorsque le problème des langues rencontré par les Zainichi est mis en question, de quelle manière la langue de leur ex-colonisateur (le japonais) est-elle représentée ? Le propos de Kim Seok-beom témoigne de la vision analogique de la théorie précédemment citée : "La langue japonaise, c'est la langue qui a violé le cerveau des embryons coréens $\gg .9$ Cette métaphore du viol par la langue japonaise suggère la genderisation au masculin de cette langue de colonisateur. Mais la relation complexe que vivent les Zainichi au sujet des langues nous permet-elle cette simple déduction tirée d'un schéma fondé sur le rapport dominant/dominé, les deux termes étant traduits respectivement par les genres du masculin et du féminin dans le contexte colonial ? Portons une attention précise sur l'expression " la langue maternelle ».

Seo Gyeong-sik nous rappelle que ce terme est déjà genré avec l'emploi du mot « la mère " incrusté dans l'expression. ${ }^{10}$ Mais s'agit-il simplement d'une question de sémantique ? N'y a-t-il pas un mouvement de la psychologie humaine plus profond dans l'emploi de cette expression, « la langue de la mère » ? Un passage du roman Sakumei de Kim Hag-yeong (1938-1985) en est un exemple allusif. ${ }^{11}$ Akiko, jeune fille zainichi (la petite sœur du protagoniste), décide elle-même de rentrer en Corée du Nord. Mais, au moment où le bateau quitte le sol japonais, Akiko délaisse le mot " eomeoni » (qui signifie « la mère » en coréen) qu'elle s’imposait d'employer, pour crier « okâsan » (qui signifie « la mère » en japonais).

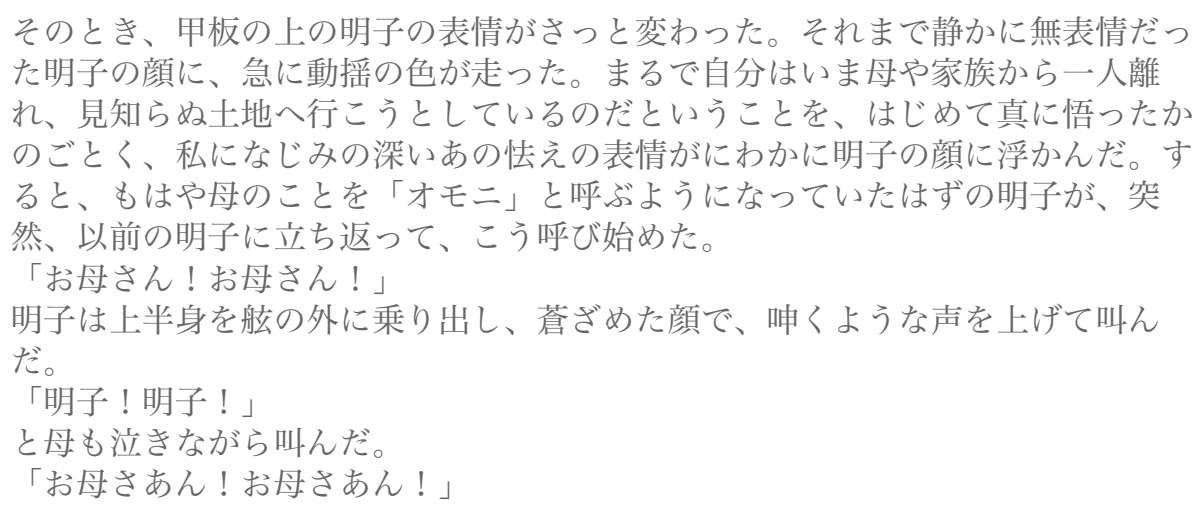


「明子お！明子お！」

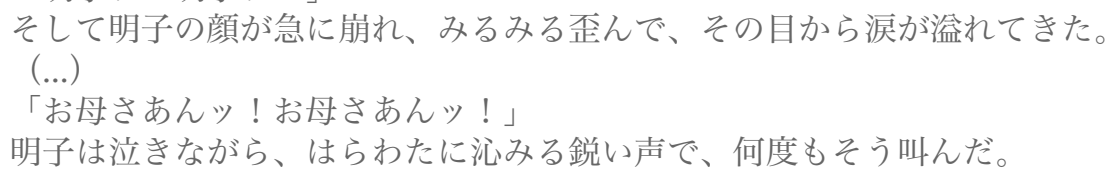

A ce moment-là, le visage d'Akiko qui était sur le pont du bateau changea brutalement. Son visage resté calme jusqu'à ce moment, ne cachait plus son trouble. Comme elle s'était rendue compte pour la première fois qu'elle allait être séparée de sa mère et de sa famille et partait seule à l'étranger, des regards apeurés apparurent sur son visage. C'était un visage familier pour moi.

Soudainement, Akiko, qui dernièrement appelait sa mère « eomeoni », se mit à crier comme si elle était redevenue Akiko d'autre fois.

«Okâsan (Maman)! Okâsan! »

En se penchant en avant, le visage tout pâle, elle criait en gémissant.

«Akiko! Akiko!»

La mère criait aussi en pleurant.

"Okâsan! Okâsan!»

«Akiko! Akiko!»

Akiko s'était effondrée, les larmes coulant de ses yeux.

(...)

«Okâsan! Okâsan!»

En pleurant, Akiko cria plusieurs fois d'une voix aigue qui pénétrait notre cœur.

C'est une scène décisive où ce personnage qui errait dans les tourments de la recherche de l'ethnicité coréenne zainichi, abandonne soudain la mère patrie, la Corée imaginaire, représentée par ce même mot " eomeoni ». Le mot japonais " okâsan » semble alors jaillir de son inconscient formé dans la langue japonaise.

Le mot qui signifie " maman », mais en coréen cette fois, apparaît également dans la scène suivante de la nouvelle de Yang Seog-il (1936-). ${ }^{12}$ Ce jeune homme de la deuxième génération zainichi, Isamu, ne maîtrise pas non plus la langue coréenne, mais il paraît indifférent à ce fait. Or, submergé par l'émotion, son inconscient le dépasse et c'est son corps qui pousse le cri, son sentiment profond, avec le mot « eomoni ».

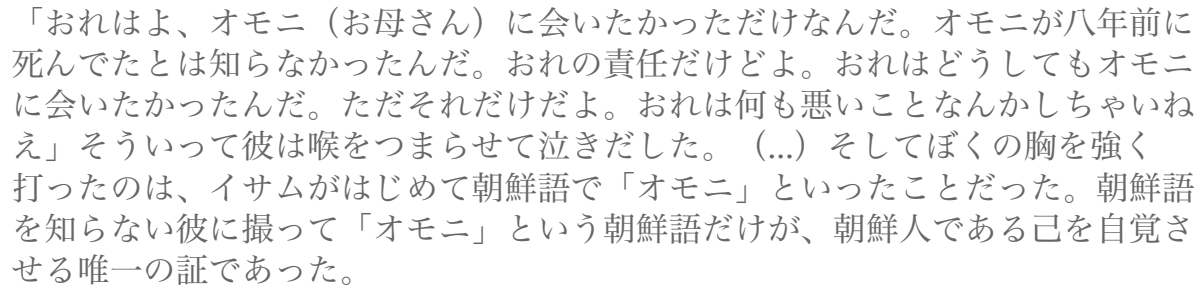

«Moi, je voulais seulement voir eomeoni (= maman). Je ne savais pas qu'elle était déjà morte il y a huit ans. Oui, c'est de ma faute de ne pas en avoir été au courant. Mais moi, je n'ai rien fait de mal ! », dit-il et il a commencé à sangloter. (...) Ce qui m'a touché profondément, c'est le mot « eomoni » prononcé pour la première fois en coréen de la bouche de Isamu. Pour lui qui ne connaît pas la langue coréenne, ce mot « eomoni » est le seul moyen de justifier sa subjectivité en tant que Coréen.

Dans ce passage, le mot « eomoni » n'est pas un simple mot coréen, mais il est décrit comme s'il faisait partie de l'existence zainichi de ce personnage d'Isamu en quête permanente de la " mère »: une mère non seulement réelle, mais aussi une mère symbolique, la langue coréenne.

9 Le contraste entre ces deux scènes nous oriente vers un aspect supplémentaire de la problématique des langues de ces jeunes Zainichi. Chez ces Zainichi, la langue liée à leur mère possède un caractère contradictoire. Elle pourrait être leur " mère ", mais non pas n'importe quelle mère. Bien que le fond de ce mot " maman ", qu'on trouve dans les deux cas, soit identique, la forme de ce mot sortant de leur bouche au moment où ces 
deux personnages sont en train de se désunir de la mère (au sens concret) est différente. Si Akiko voyait jusqu'alors la langue coréenne comme sa véritable mère métaphorique qui l'a abandonnée, Isamu est lancé désespérément dans la quête de cette mère qui doit exister quelque part. En faisant référence à la théorie freudienne liée à la littérature, nous pourrions considérer Akiko comme " enfant retrouvé » et Isamu, comme "enfant bâtard ». ${ }^{13}$

Mais nous rencontrons ici une contradiction primaire : l'origine biologique de l'enfant est toujours assurée par sa mère. ${ }^{14}$ Autrement dit, comme Freud l'affirme, repris ensuite par Marthe Robert et Julia Kristeva, l'illégitimité de l'enfant bâtard n'a pas d'autre fondement que l'adultère supposé de la mère. ${ }^{15}$ Or, dans le cas de ces enfants Zainichi, c'est leur mère symbolique, c'est-à-dire, cette« langue maternelle », qui est problématique. Car c'est cette mère, et non pas le père comme chez Freud, qui est « l'origine » de l'illégitimité de l'enfant. Certains Zainichi voient la langue coréenne comme leur langue maternelle, chez d'autres c'est la langue japonaise. Faisons alors référence au doute de Jacques Derrida : dans sa critique de la vision de la langue maternelle chez Hannah Arendt, il souligne la remplaçabilité de cette mère conceptuelle. ${ }^{16} \mathrm{Si}$ nous établissons un lien entre ces langues (des Zainichi) et cette appellation de " la langue de la mère », terme métaphoriquement genré au féminin, nous pouvons dire que les Zainichi possèdent plus qu'une mère. Malgré son existence incertaine, la langue coréenne qui forme le fondement existentiel dans leur vision conceptuelle de la langue dite maternelle est du genre féminin, tandis que la langue japonaise qui possède le pouvoir de domination issue de la colonisation japonaise est du genre masculin. Mais il s'agit maintenant d'analyser deux aspects parfois contradictoire et parfois complémentaire de la mère, en nous référant à l'idée lacanienne de « la mère phallique » et de la mère en tant que « l'abject».

D'un côté, nous pourrions considérer la langue maternelle en tant que la mère des Zainichi comme une mère qui est fantasmatiquement pourvue d'un phallus, symbole de la domination masculine : " la mère phallique ». ${ }^{17}$ Les concepts de mère phallique et de mère castratricesont déjà présenté la fin des années 1940 chez certains psychanalystes : Il a vu la castration par la mère bien plus souvent que par le père à tel point qu'il en est presque arrivé à nier la crainte de la castration par le père. ${ }^{18}$ L'affirmation suivante de Lee Yang-ji (1955-1992), écrivain femme de la deuxième génération zainichi, concernant sa langue maternelle (=le japonais) décrit ce trait de la mère phallique : « La langue maternelle, c'est-à-dire, la langue que j'entendais de ma mère dans mon enfance, reste collée au fond de mes oreilles. Et elle joue un rôle décisif, elle règne dans mes pensées et domine mon existence d'une manière violente $»{ }^{19}$ Chez elle, la langue maternelle japonaise est représentée par une image de la mère possédant d'un phallus symbolique qui règne comme la Loi paternelle absolue chez l'enfant.

D'un autre côté survient une autre image de la mère, en tant que « l'abject ». Lors de la différenciation instinctive au stade préoedipien, l'enfant fait tous ses efforts pour rompre avec la «mère archaïque». Cet événement psychique dans le monde de l'Imaginaire donne le néologisme « l'abject » (Kristeva $1982: 17) .{ }^{20}$ Le comportement de l'enfant visà-vis de l'abject comprend, selon certains courants psychanalytiques, toutes les vicissitudes de l'amour et de la haine. ${ }^{21}$ Dans la théorie féministe "gynesis », ce terme est décrit ainsi : «l'abject se conçoit, de manière générale, comme ce qui est digne du plus grand mépris, qui inspire une violente répulsion, le dégoût alimentaire en étant la forme la plus élémentaire " (nous soulignons). ${ }^{22}$ Afin de compléter la description de l'abject dans les œuvres de la littérature zainichi, nous citons le passage suivant.

ひとりの部屋で包みを開け、ハンバーガーにかぶりついた瞬間、突然吐き気をお ぼえた。それでもどうにか咀嚼はしたが、吞み下すことができずに結局口の中の ものをティッシュの上に吐き出した。 
ひと口だけ觢った跡のついたハンバーガーを、雅美は呆然と見つめた。吐き気に 襲われる前に鼻をついた匂いが何であるかは明確に判っていた。

ニンニクの匂いだった。それを嗅いだのと同時に、なぜか雅美の頭の中を満員の

地下鉄車内の光景がよぎっていた。

Seule dans la chambre, elle a enlevé l'emballage du hamburger. Mais, à peine a-telle croqué qu'elle a la nausée. Bien qu'elle essaie de mâcher, elle n'arrive pas à l'avaler, et recrache tout dans le kleenex.

Sous le choc, Masami regardait avec stupéfaction ce hamburger imprimé de la marque de ses dents. Elle identifiait très bien l'odeur qu'elle sentait juste avant la sensation de nausée. C'était l'odeur de l'ail. Cette odeur a suscité immédiatement en elle, sans savoir pourquoi, une scène dans le métro bondé.

Le corps de cette jeune Zainichi ne peut pas s'empêcher de cracher tout ce qui lui rappelle la vie quotidienne de la péninsule et la langue parlée de cet endroit. ${ }^{23}$ Les contacts avec sa colocataire, surtout les repas partagés avec cette jeune femme coréenne très bavarde et nonchalante, deviennent également des moments insupportables pour elle, qui devient incapable d'avaler la moindre nourriture. Chez ce protagoniste, ce trouble corporel (l'anorexie) est suscité par l'apprentissage obsessionnel de la langue qui devrait être sa langue maternelle, le coréen. Cette langue ainsi décrite fait un contraste avec la vision de la langue japonaise en tant que la mère phallique chez Lee Yang-ji. Car, dans ce roman autobiographique de Sagisawa Megumu (1968-2004), la langue coréenne est une métaphore de la mère en tant que « l'abject ». La description de ces deux aspects à la fois contradictoire et complémentaire, nous montre clairement de quelle manière ces Zainichi sont prisonniers de cette langue imaginée maternelle, du concept de cette langue.

\section{Le mythe de la langue maternelle}

Comme nous devons le constater, certains jeunes Zainichi tentent de se réapproprier ce qu'ils estiment être la langue de leur vraie mère, donc leur vraie langue maternelle, le coréen. Là, nous voyons apparaître une contradiction : c'est-à-dire, premièrement, que tous leurs efforts pour apprendre la « langue maternelle » se mettent en contradiction avec le consensus selon lequel une langue appelée maternelle correspond à la langue parlée par la majorité des gens dans le pays où ils sont nés et devrait ainsi s'apprendre quasi-naturellement, sans peine. Et deuxièmement, ces jeunes Zainichi n'ont jamais " possédé » la langue coréenne, si bien que l'expression se « ré-approprier » cette langue est contradictoire. Pour les Zainichi à partir de la deuxième génération, la langue dite « maternelle » est la langue japonaise, tandis que la langue coréenne est une langue « non-maternelle ». Comment pourrions-nous ainsi interpréter leur souhait le plus cher de réappropriation de cette langue coréenne en tant que leur « véritable langue maternelle »? En second lieu, il nous faut donc questionner ce concept de la langue maternelle lui-même.

Des phénomènes et mouvements symétriques de réappropriation de la langue coréenne et de refus de la langue japonaise, nous pourrions dégager le trait suivant : chacune de ces deux langues, le japonais et le coréen, n'est conçue et imaginée qu'en tant que reflet de l'autre. Autrement dit, pour les jeunes Zainichi il existe malgré eux une langue " maternelle » (le japonais) qui s'oppose à une autre langue qui, elle, est censée être la langue ethnique (et nationale chez certains), le coréen. Ces constructions fantasmées chez les jeunes Zainichi sont illustrées dans un passage du roman Koku (Temps) de Lee Yang-ji. ${ }^{24}$ En s'efforçant de devenir " coréenne le plus vite possible », le protagoniste (une jeune femme Zainichi) part en Corée apprendre la langue coréenne. 
ソンセンニム、私たちは、在日同胞です。日本で生まれ育ち、日本語ばかりにと りかこまれて生きてきた者たちです。日々、同化と風化を強いられる環境の中に いて、私たちは民族的主体性を確立できないまま、悶々としてきました。ここに いる学生たちは、それぞれが、さまざまな動機を持って、母国留学を決意しまし た。しかし、ただ一点、ウリマル（母語）を学ばなければならないのだ、という 熱い思いは共通しています。

\begin{abstract}
Maître, nous sommes expatriés Zainichi. Nous sommes nés au Japon et vivions entourés uniquement par la langue japonaise. Dans ces circonstances qui nous imposaient l'assimilation au Japon et l'effritement de l'ethnicité coréenne, nous souffrions du manque de subjectivité ethnique. Chacun de nous, qui se trouve ici aujourd'hui, a décidé de faire ses études dans notre patrie, en Corée, grâce au programme d'échange. Les motivations de cette décision sont sans doute diverses. Mais, tous nous partageons une passion et sentons la nécessité (prioritaire) d'apprendre notre langue maternelle, le coréen.
\end{abstract}

Selon la linguiste Marina Yaguello, on possède une langue interne, qui ne peut être remplacée par une autre acquise ultérieurement. ${ }^{25}$ Cette vision de la langue maternelle véhicule le consensus selon lequel la langue maternelle ne s'apprend pas comme on apprend d'autres langues. Or, si un tel consensus était valable, la constatation de notre protagoniste pour qui « apprendre notre langue maternelle » se fait avec beaucoup de peine, dévoile ironiquement la contradiction profonde des Zainichi vis-à-vis de cette langue.

De nombreuses études démontrent que la langue japonaise moderne en tant que langue nationale a été créée en même temps que l'État-Nation sous l'expansion coloniale du Japon. ${ }^{26}$ Comme Sakai Naoki le fait remarquer, avant le 19ème siècle au Japon, la langue maternelle d'un individu ne signifiait pas sa langue nationale naturelle. A la fin du 19 ème siècle, l'harmonisation entre l'oral et l'écrit procède d'un détachement vis-à-vis des langues écrites universelles (ici, celle de l'empire chinois) indépendantes de la phonétique et transcendantes, donnant ainsi naissance à la langue nationale. ${ }^{27}$ Selon Sakai, c'est à cette époque que la figure de la langue maternelle japonaise en tant que langue nationale naturelle a été créée, au travers d'un mécanisme de la cofiguration. ${ }^{28}$ Sakai redéfinit " la langue maternelle » ainsi : l'existence elle-même de la langue maternelle ne peut être linguistiquement thématisée car elle précède toutes autres déterminations prédicatives, elle est le néant. ${ }^{29}$ De ce point de vue, Sakai affirme que la relation avec notre langue maternelle avec laquelle nous pensons exprimer immédiatement notre intériorité ne serait qu'imaginaire, un fantasme de la communion. ${ }^{30}$

Revenons sur Yu-hui de Lee Yang-ji. Après avoir passé un certain temps en Corée métropolitaine, le protagoniste Zainichi se rend compte que sa sensibilité et sa pensée ne sont compatibles qu'avec la langue japonaise. La présence de cette langue qu'il voit comme sa langue maternelle suscite en lui une sorte de culpabilité. Mais une telle interprétation du protagoniste n'est-elle pas déjà prise dans le mythe de la langue maternelle ? Ne glisse-t-elle pas par la suite facilement vers le discours de l'identité basée sur l'unité linguistique ${ }^{31}$ La réaction de Yu-hui qui se voit comme damnée par sa langue maternelle japonaise nous expose le piège créé, dans le contexte moderne, par l'idéologie de la langue de l'Etat-nation.

La jeune femme Zainichi protagoniste de Koku de Lee Yang-ji affirme qu'elle ne pourra pas être libérée de la langue japonaise, avant de se libérer de la langue coréenne. ${ }^{32}$ Une telle contestation contraste significativement avec le propos précédemment cité de Kim Si-jong : leur vision de ces deux langues fait un parallèle avec le cas de deux protagonistes, celui de Lee Yang-ji et celui de Sagisawa que nous avons précédemment cités. Ici encore nous apercevons l'apparition du concept de la langue maternelle et le schéma de la domination phallocentrique de cette langue. La difficulté de détacher ces 
deux conceptions se manifeste encore plus clairement dans le domaine de la littérature formée parallèlement à la modernité. Ceux qui écrivent, les zainichi y compris, ne peuvent pas s'échapper de ce contexte historico-politique. Comme Karatani Kôjin l'affirme, « la nation, c'est la littérature moderne ».33

Ce que l'on appelle ici « notre intérieur » peut-être mis en lien avec la réflexion de Karatani : "l'intériorité » est découverte non seulement dans la littérature japonaise moderne mais aussi «par » cette littérature.En considérantl'homme dans l'institution chrétienne occidentale comme "bête d'aveu ", Michel Foucault nous suggère également cette intériorité de chaque individu comme une invention moderne. ${ }^{34}$ Le monologue du protagoniste de Koku de Lee Yang-ji nous permet d'apercevoir l'artificialité de ce qu'on appelle « notre intérieur ».

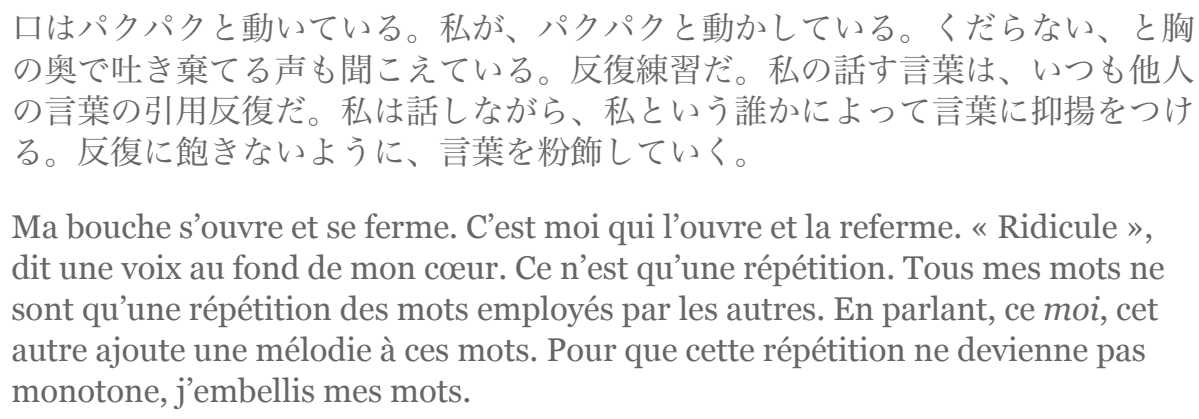

\section{L'impossibilité de s'exprimer}

En troisième lieu, tournons nous vers une forme que prend dans certaines œuvres littéraires la question de la langue " maternelle » des Zainichi : l'impossibilité de s'exprimer. Yu-hui, jeune femme Zainichi souffre du sentiment de culpabilité de ne pas pouvoir aimer la langue coréenne, qui devait être sa langue maternelle. L'héroïne est ainsi confrontée à l'état d'aphasie. 35

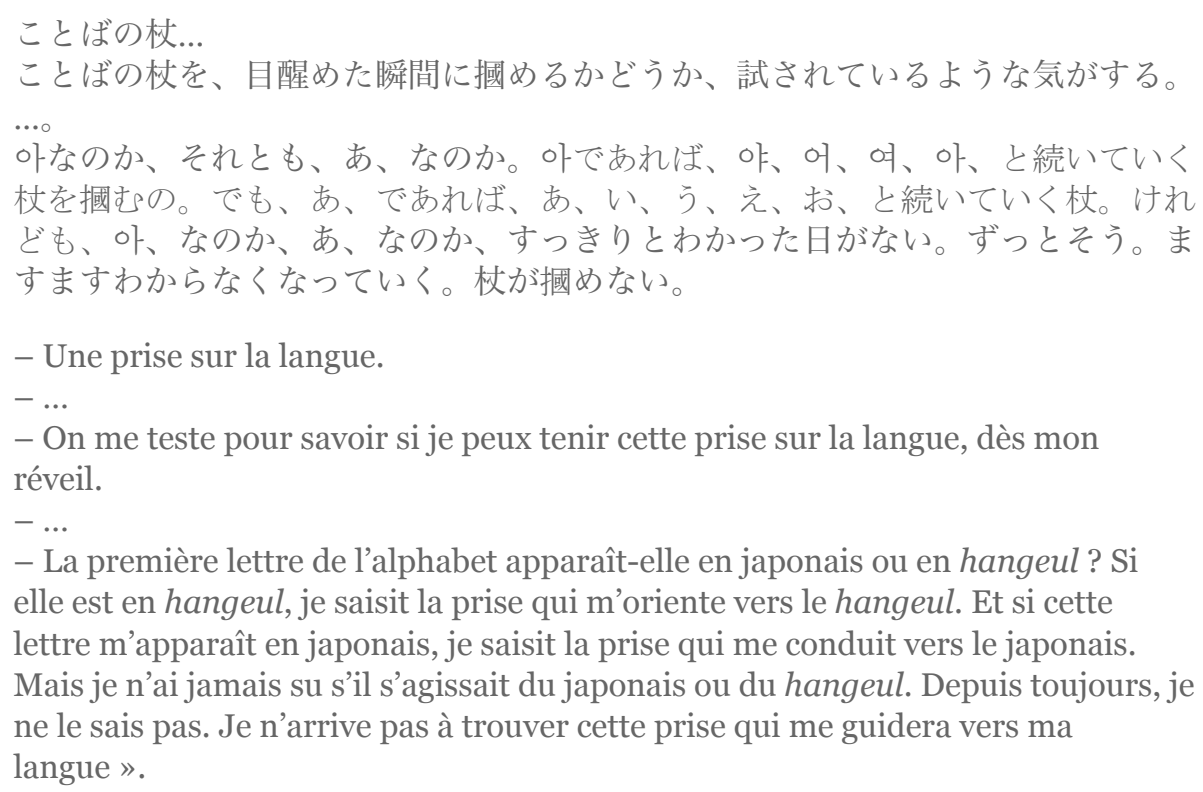

Dans ce passage de Yu-hui, ni le japonais qu'elle croyait jusqu'alors sa langue maternelle, ni le coréen, la langue qu'elle rêvait de posséder comme sa "vraie » langue maternelle, ne permettent à cette jeune femme Zainichi de s'exprimer. On pourrait entrevoir ici, dans la description de cet état d'aphasie, l'objection de Sakai faite à cette illusion de la langue maternelle souvent considérée comme une substance réelle qui 
existe incontestablement. ${ }^{36}$

A côté de cette absence de langue (l'aphasie), deux femmes écrivains Lee Yang-ji et Sagisawa décrivent également le jaillissement de mots qui ne prennent pas encore de forme. Cette manifestation nous suggère l'existence d'une autre langue pour les Zainichi, une langue qui n'est ni la langue nationale japonaise, ni la langue ethnique coréenne, ces langues institutionnelles, langues de la communauté nationale imaginée.

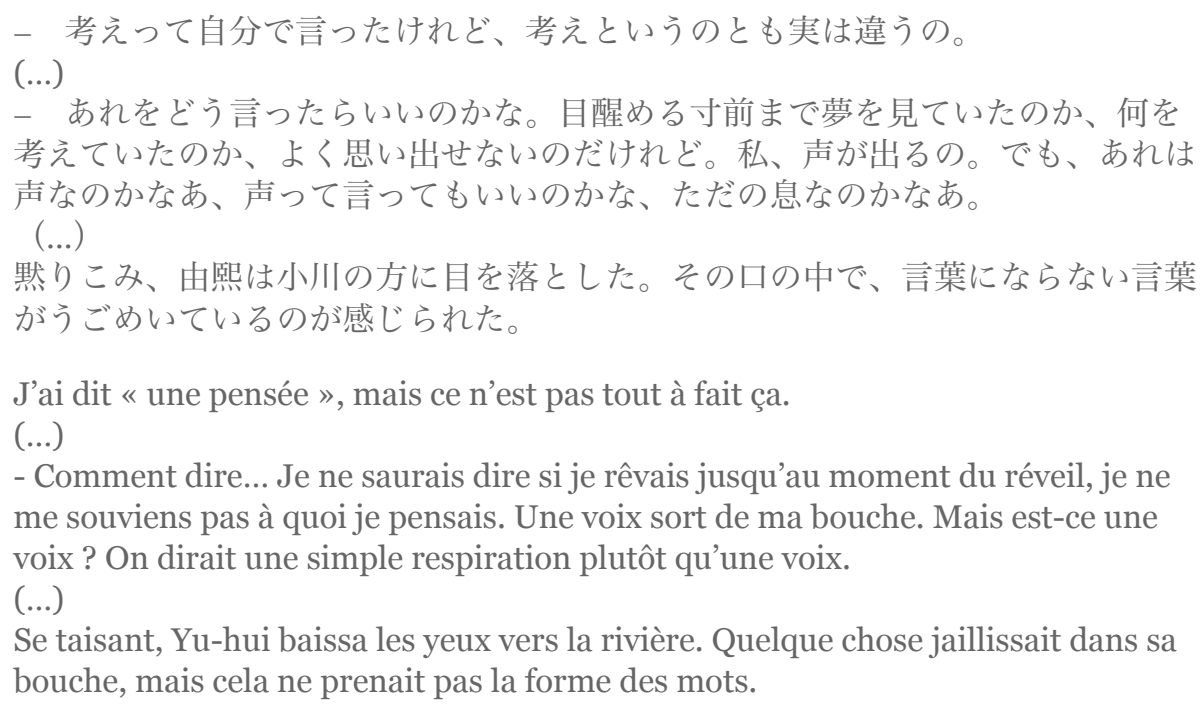

De même dans le roman de Sagisawa se trouve ce jaillissement des mots, échappant à toute description, au moment où le protagoniste, Masami, essaie de répondre à la question posée par son ami-camarade zainichi lorsqu'elle quitte le sol coréen après son séjour linguistique éprouvant : «Après tout, aimes-tu ce pays (= la Corée) ? »37

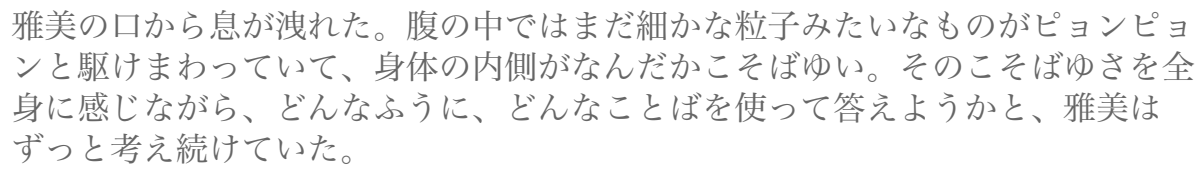

Cette sensation de jaillissement décrite par Lee Yang-ji et Sagisawa consiste en un moment décisif où les jeunes femmes zainichi sont jetées pleinement dans les batailles existentielles et dans la formation de soi, en tant que sujet parlant. Ce « jaillissement des mots » (Yu-hui), ces « petits grains non identifiables » (Masami), ne se rapprocheraientil pas du mouvement du magma, nommé « le sémiotique » par Julia Kristeva ? Cette langue, ou plutôt quelque chose qui ne prend pas encore de forme, chez nos protagonistes Zainichi (Yu-hui et Masami) n'est soumis au règne ni du japonais, ni du coréen. Nous pouvons considérer ce phénomène non-langagier, sorte de rythme corporel, fourmillement non-structuré, comme un générateur de la langue.

\section{Conclusion}

Kristeva compare ce " sémiotique » au terme de chora chez Platon et précise : " Nous empruntons le terme de chora à Platon dans le Timée pour désigner une articulation toute provisoire, essentiellement mobile, constituée de mouvements et de leurs stases éphémères ». Il s'agit d'une «espace matriciel, nourricier, innommable, antérieur à l'Un, 
à Dieu, et par conséquent, défiant la métaphysique» (Kristeva 1979 : 7). ${ }^{38}$ Le " sémiotique ", ce générateur de la langue pourrait correspondre à " la langue maternelle » définie par Sakai, celle qui précède toutes les autres déterminations prédicatives. ${ }^{39}$ Dans les œuvres de Lee Yang-ji et de Sagisawa que nous avons précédemment citées, ce générateur non identifiable est présenté comme une sorte d'impossibilité de s'exprimer. Et, justement, ne serait-ce pas cette impossibilité qui est la tâche de la littérature ? Nous pourrons ainsi dire : comment la littérature peut-elle être le lieu même de cet impossible?

\section{Notes}

1 Isogai Jirô 磯貝治良, «Zainichi » bungakuron〈在日〉文学論 (Études sur la littérature zainichi), Tôkyô, Shinkansha, 2004, p.12.

2 Jang a choisi une revue japonaise parce qu'à cette époque le monde des lettres était plus ouvert au Japon métropolitain que dans la péninsule coréenne. Jang éprouvait le fort désir de devenir romancier ; ajouter à cela que le sujet de son premier roman traitait des faits réels de la colonie japonaise -la péninsule coréenne- et répondait parfaitement à la demande du monde des lettres japonais qui cherchait un sujet original. C'est ainsi que Jang a fait ses débuts dans une revue japonaise : on ne peut en déduire qu'il ait renié ses racines coréennes. Vers la fin de sa carrière, il écrivit également un roman en anglais. Voir : Tsukubadaigaku Bunkahihyô Kenkyûkai (Dir.) 筑波大学文化批評研究会編, Shokuminchishugi to ajia no hyôshô 植民地主義とアジアの表 象 (Colonialisme et représentation de l'Asie), Tsukuba, Tsukubadaigaku Bunkahihyô Kenkyûkai, 1999. Voir : Hayashi Kôji 林浩冶, Zainichichôsenjin nihongobungakuron 在日朝鮮人日本語文学 論 (Études sur la littérature zainichi de langue japonaise), Tôkyô, Shinkansha, 1991; Chôsen wo shiru jiten 朝鮮を知る事典 (Encyclopédie pour connaître le Chosǒn), Tôkyô, Heibonsha, 1986. Quant à Kim Sa-ryang, il a publié quelques nouvelles dans les revues japonaises.

Ex) « Hikari no naka ni 光の中に», Bungêshuto 文芸首都, 1939.10. ; 《Tenma 天馬 », Bungêsshunjû 文藝春秋, 1940.06.

3 Kim Seok-beom 金石範, Kotoba no jubakuことばの呪縛 (Ensorcellement des langues), Tôkyô, Chikumashobô, 1972.

$4 \mathrm{Kim}$ Si-jong 金時鐘, «Watashi no naka no nihon to nihongo 私の中の日本と日本語 (Le Japon et le japonais en moi) » in Kim Si-jong no shi : mou hitotsu no nihongo金時鐘の詩 : もう一つの 日本語 (Les poèmes de Kim Si-jong : un autre japonais), Mozukôbô, 2000, p.214.

5 Kim Huna 金壎我, Zainichichôsenjin josei bungakuron 在日朝鮮人女性文学論 (Études sur la littérature féminine zainichi), Tôkyô, Sakuhinsha, 2004.

6 Ôgoshi Aiko 大越愛子 et Igeta Midori 井桁碧 (Dir.), Sengoshisô no politikusu : Sengo, Bôryoku, Jendâ 戦後思想のポリティクス-戦後・暴力・ジェンダー (Politique de la pensée d’après-guerre : après guerre, violence, genre), Tôkyô, Seikyûsha, 2005, p.267.

7 Sakai Naoki 酒井直樹, « Eizô to gendâ : eiga no naka no renai to jikodoitsusei no ryûdôsei 映像 とジェンダー：映画の中の恋愛と自己同一性の流動性 (la relation amoureuse dans le cinéma et la fluidité de l'identité) » in Keizoku suru shokuminchi shugi : gendâ, minzoku, jinshu, kaikyû 継続する植民地主義：ジェンダー／民族／人種／階級 (Colonialisme continu : genre, ethnie, race, classe), Tôkyô, Seikyûsha, 2005, p.277.

8 Kang Sang-jung 姜尚中, Orientarizumu no kanata e : kindai bunka hihan オリエンタリズム の彼方 - 近代文化批判 - (Au delà de l'orientalisme : critique sur la culture moderne), Tôkyô, Iwanamishoten, 2004, pp.105-106.

9 Kim Seok-beom 金石範, Minzoku, kotoba, bungaku 民族・ことば・文学 (Ethnie, langue, littérature), Tôkyô, Sôjusha, 1976, p.13.

10 Seo Gyeong-sik 徐京植, «Bogo to Bokokugo no sôkoku : Zainichichôsenjin no gengokeiken 母語と母国語の相克-在日朝鮮人の言語経験 (Conflit entre la langue maternelle et langue nationale : expérience linguistique chez les Zainichi) », Tôkyôkeizaidaigaku jinbunshizenkagaku ronshû No.126, 2008, p.54.

11 Extrait de Sakumei in Takeda Seiji 竹田青嗣, Zainichi to iu konkyo 在日という根拠 (Zainichi : fondements), 1983, pp.179-181.

12 Yang Seog-il 梁石日, « Kureijî Hôsu I クレージーホース I (Crazy Horse I) » in Takushî kyôsôkyoku タクシー狂躁曲 (Rhapsodie du taxi), Tôkyô, Chikumashobô, 1987, p.191. 
13 Marthe Robert, Roman des origines et origines du roman, Paris, Gallimard, 1972.

14 « Je pense que dans l'imaginaire, la continuité maternelle est ce qui garantit l'identité ». Propos de Julia Kristeva, « Entretien avec Julia Kristeva » in Les Cahiers du GRIF, N. 32, 1985, p.23.

15 Marthe Robert, Roman des origines et origines du roman, Paris, Gallimard, 1972, p.55.

16 Jacques Derrida, Tatta hitotsu no, watashinomono dewanai gengo : tasha no tan'itsugengo shiyô たつた一つの、私のものではない言語：他者の単一言語使用 (Le monolinguisme de l'autre ou la prothèse d'origine), Iwanamishoten, 2001, p.60. Derrida remet en question également le consensus se trouvant dans la théorie freudienne de la légitimité de la naissance venue de la mère. En rejetant, sur tous les plans, l'Un absolu, il rejette aussi la mère biologique seule légitime et suggère la pluralité de la " mère » en citant les cas de la mère porteuse, de l'insémination artificielle, .... idem, p.160.

17 Laplanche et J.B Pontalis, Vocabulaire de la Psychanalyse, Presses Universitaires de France, Paris, 1976.

18 James-H.Leuba, « Mère phallique et mère castratrice » in Revue Française de Psychanalyse, avril-juin 1949, tome XIII, $\mathrm{n}^{\circ}$ 2, p. 317.

19 Lee Yang-ji李良枝, Lee Yang-ji zenshû『李良枝全集』(Euvres complètes de Lee Yang-ji), Kôdansha, 1993, p.663.

20 Morny Joy, « Et la chair s'est faite verbe » in Recherches féministes, vol. $3 n^{\circ}$ 2, 1990, p. 118.

21 Morny Joy, idem, p.118.

22 Le mot «gynesis » est formé sur « Genèse », il signifie la féminité refoulée par le système phallocentrique dans certains courants de féminisme. Amélie Coulombe-Boulet, « L'Abjection dans L'ingratitude de Ying Chen : parcours d'une dévoration identitaire » in Posture No.5 : voix de femmes de la francophonie, printemps 2003, p.68. ; Soejima Miyuki 副島美由紀, « Tenshi to saibôgu no aida »「使とサイボーグのあいだ」 (Entre l'ange et le cyborg) inKareidosucôpu『カレイドスコープ:中村志郎先生退官記念論集』(Kaléidoscope : numéro spécial pour la retraite du professeur Nakamura Shirô), 1996, p.385.

23 Sagisawa Megumu 鷺沢萌, Kimi wa kono kuni o suki ka 君はこの国を好きか (Aimes-tu ce pays ?), Tôkyô, Shinchôsha, 1997, p.159.

24 Lee Yang-ji 李良枝, Koku 刻 (Temps), 1984, p.6o.

25 Marina Yaguello, Catalogue des idées reçues sur la langue, Paris, Seuil, 1988, p.81.

26 Matsumoto Ken’ichi 松本健一, «Nihongo to û aidentiti 日本語というアイデンティティ (L'identité la langue japonaise) », Daikôkai No.46 p.174; Fukuma Yoshiaki 福間良明, Henkyô ni utsuru Nihon 辺境に映る日本 (Le Japon reflété par ses frontières), Tôkyô, Kashiwashobô, 2003.

27 Karatani Kôjin 柄谷行人, Nihonseishinbunseki 日本精神分析 (Psychanalyse du Japon), Tôkyô, Kôdansha, 2010. De même, Anderson cite le latin, le pali, l'arabe littéraire et le chinois littéraire comme des langues sacrées des communautés classiques.Benedict Anderson, Zôho sôzô no kyôdôtai : nationalism no kigen to ryûkô 増補・想像の共同体ーナショナリズムの起源 $と$ 流行 (L'Imaginaire national: réflexions sur l'origine et l'essor du nationalisme), trad. de l'anglais par Shiraishi Saya 白石さや, Shiraishi Takashi 白石 隆, Tôkyô, NHK shuppankabushikigaisha, 2002, p.36.

28 Sakai Naoki, Translation and subjectivity, University of Minnesota Press, Minneapolis, London, 1997, p.21.

29 Sakai Naoki, Translation and subjectivity, p.21.

30 Sakai Naoki, « Nationality and the Politics of the Mother Tongue », in Deconstructing Nationality, New York, CEASBooks, 2005, p.19.

31 Sakai Naoki, Translation and subjectivity, p.20.

32 Lee Yang-ji 李良枝, Koku 刻 (Temps), 1985, Tôkyô, Kôdansha, p.178.

33 Karatani Kôjin 柄谷行人, Nihon seishinbunseki 日本精神分析 (Psychanalyse du Japon), Tôkyô, Bungeishunjû, 2002, p.12.

34 Karatani Kôjin, Nihon kindaibungaku no kigen日本近代文学の起源 (Les origines de la littérature japonaise moderne), Tôkyô, Iwanamishoten, 1998 ; Michel Foucault, Histoire de la sexualité, tome 1 : La Volonté de savoir (chap.3), Paris, Gallimard, 1994.

35 Lee Yang-ji 李良枝, Yu-hui 由熙 (Yu-hui), Tôkyô, Kôdansha,1989, pp.122-123.

36 Sakai Naoki 酒井直樹, Shizan sareru nihongo nihonjin死産される日本語・日本人 (Le 
japonais et les Japonais mort-nés),Shinyôsha, 2001, pp.169-170.

37 Sagisawa Megumu 鷺沢萌, Kimi wa kono kuni o suki ka 君はこの国を好きか (Aimes-tu ce pays ?), Tôkyô, Shinchôsha, 1997, p.250.

38 Morny Joy, «Et la chair s'est faite verbe » in Recherches féministes, vol. $3 n^{\circ}$ 2, 1990, p. 114.

39 Sakai Naoki 酒井直樹, Nihonshisô toiu mondai 日本思想という問題：翻訳と主体 (Translation and Subjectivity: On Japan and cultural nationalism), 1997, p.215.

\section{Pour citer cet article}

Référence électronique

Ayame HOSOI, "La langue japonaise est-elle la « mère » des Zainichi ? », Transtext(e)s

Transcultures 跨文本跨文化 [En ligne], 8 | 2013, mis en ligne le 02 décembre 2013, consulté le 16 janvier 2014. URL : http://transtexts.revues.org/490

\section{Auteur}

\section{Ayame HOSOI}

Ayame HOSOl est maître de conférences en études japonaises à l'Université Jean Moulin (Lyon) et membre de l'IETT (Institut d'Études Transtextuelles et Transculturelles). Son travail s'articule autour de la littérature japonaise contemporaine et sur les questions de l'Asie et ses diasporas.

Ayame HOSOI is Assistant Professor in Japanese Studies at the Jean Moulin University (Lyon) and a member of the IETT (Institute for Transtextual and Transcultural Studies). Her work focuses on contemporary Japanese literature and Studies of Asia and its Diasporas.

\section{Droits d'auteur}

(C) Tous droits réservés 